\title{
Trends in Cell Biology
}

\section{Stitching organelles: Organization and function of specialized plant membrane contact sites \\ --Manuscript Draft--}

\begin{tabular}{|c|c|}
\hline Manuscript Number: & TCB-D-16-00039R1 \\
\hline Corresponding Author: & $\begin{array}{l}\text { Abel Rosado } \\
\text { University of British Columbia } \\
\text { Vancouver, BC CANADA }\end{array}$ \\
\hline First Author: & Jessica Pérez-Sancho \\
\hline & Jens Tilsner \\
\hline & A. Lacey Samuels \\
\hline & Miguel A. Botella \\
\hline Abstract: & $\begin{array}{l}\text { The coordination of multiple metabolic activities in plants relies on an inter-organelle } \\
\text { communication network established through membrane contact sites (MCS). The MCS } \\
\text { are maintained in transient or durable configurations by tethering structures, which } \\
\text { keep the two membranes in close proximity, and create chemical micro-domains that } \\
\text { allow localized and targeted exchange of small molecules and possibly proteins. The } \\
\text { last few years have witnessed a dramatic increase in our understanding of the } \\
\text { structural and molecular organization of plant inter-organelle MCS, and their critical } \\
\text { roles in plant specialized functions including stress responses, cell to cell } \\
\text { communication and lipid transport. In this review, we summarize recent advances in } \\
\text { understanding the molecular components, structural organization, and functions of } \\
\text { different plant-specific MCS architectures. }\end{array}$ \\
\hline
\end{tabular}




\section{Trend Box}

1. Inter-organelle communication in plants relies on membrane contact sites (MCS), specialized membrane junctions that facilitate molecular exchanges between apposed bilayers.

2. The transfer of molecules between plant organelles uses both evolutionarily conserved eukaryotic MCS and also plant-specific MCS with unique architecture and specialized functions.

3. MCS establishment and function is precisely regulated by protein tethering complexes. These components have been extensively studied in yeast and mammals but until recently no MCS tethers have been identified and functionally characterized in plants.

4. The functional characterization of plant-specific membrane contact sites highlights their essential roles in processes not described for other eukaryotic organisms such as tissue development, intercellular trafficking, and stress responses. 
1 Stitching organelles: Organization and function of specialized plant 2 membrane contact sites

Correspondence should be addressed to: abel.rosado@botany.ubc.ca Phone: +1 604-827-1662

\section{Abstract} communication network established through membrane contact sites (MCS). The MCS are maintained in transient or durable configurations by tethering structures, which keep the two membranes in close proximity, and create chemical micro-domains that allow localized and targeted exchange of small molecules and possibly proteins. The last few years have witnessed a dramatic increase in our understanding of the structural and molecular organization of plant inter-organelle MCS, and their critical roles in plant specialized functions including stress responses, cell to cell communication and lipid transport. In this review, we summarize recent

27 advances in understanding the molecular components, structural organization, and functions of 28 different plant-specific MCS architectures. 


\section{Plant MCS: Specialization of evolutionarily conserved communication 2 nodes}

A hallmark of plant cellular organization is a sophisticated subcellular compartmentation system that includes the nucleus, the endomembrane system, and organelles derived from endosymbiotic associations including mitochondria and plastids. While subcellular compartmentation enables the efficient segregation of complex biochemical processes, it also imposes a physical barrier impeding the free flux of metabolites and macromolecules between organelles [1]. To circumvent this limitation, plant cells exploit evolutionarily conserved structures known as membrane contact sites (MCS) that establish physical interactions and enable non-vesicular transfer of molecules between apposed organelle membranes [2]. MCS hallmarks have been extensively described in different eukaryotic organisms and their most prominent characteristics can be summarized as i) MCS are tight junctions (Box 1) where two organelle membranes are tethered via protein complexes ii) MCS organelle membranes do not fuse (although hemi-fusion intermediates may be possible) iii) MCS are enriched in specific proteins and/or lipids and iv) MCS segregate and/or regulate trafficking and signalling events [25].

It is likely that plant MCS provide highly specialized microenvironments with conserved functions similar to other eukaryotic organisms. These functions include inter-organelle lipid transfer (ER-mitochondria, ER-Golgi, and Nuclear-Vacuole junctions), organelle retention and transport (ER-peroxisome junctions), and $\mathrm{Ca}^{2+}$ homeostasis regulation (ER-PM junctions). The evidence for these conserved functions has been extensively reviewed recently in yeast and mammals [2-8]), therefore the focus of this review is the role of plant MCS in the coordination of multiple plant-specific processes including, but not restricted to, the regulation of environmental stress and developmental responses [9-11], intercellular communication [12-13], the maintenance of lipid homeostasis at chloroplast-ER junctions [14-15], and the generation of stress-inducible responses between plastids and different organelles including nucleus, ER, mitochondria and peroxisomes [16-18]. 
Because most plant cells have cell walls and a large central vacuole, and many metabolic functions are carried out by different types of plastids, plant MCS have adopted unique features such as tissue-dependent associations between the cortical cytoskeleton and the cortical ER that, in turn, determine the ER-PM contact sites localization [9] (Figure 1, Box 2). Plant MCS have also maintained specialized architectures, such as plasmodesmata [19] and chloroplast-ER contact sites [20] reflecting their unique origin and functional specialization. In this review we describe our current knowledge of the molecular components, structural bases and functional specialization of these plant-specific inter-organelle arrangements (Figure 2A). We also discuss current challenges and future directions we envision for plant MCS research.

\section{The search for plant MCS components: Not all eukaryotes are equal}

Most of our current knowledge regarding plant MCS derives from direct visualization of MCS structures (BOX2), and the identification and functional characterization of MCS components bridging the juxtaposed membranes, providing physical stability to the adjacent organelles, and promoting the exchange of molecules between them [2-5]. Plant MCS research has benefited from the extensive characterizations of close homologs and evolutionarily conserved MCS components in other eukaryotic systems. For example, homology searches using known yeast and mammalian MCS components as baits, were instrumental for the identification of two families of tethering components, the Vesicle-associated membrane protein (VAMP)associated proteins (VAPs) [10, 21], and the synaptotagmins (SYTs) [9] in plants. prominent feature is an N-terminal major sperm protein (MSP) protein-protein interaction

22 domain [22] (Figure 3A). In mammals, a number of VAP interacting proteins are involved in lipid 23 transfer and contain phenylalanines in an acidic tract (FFAT) and/or the sterol-binding 24 steroidogenic acute regulatory (StAR)-related lipid transfer (StART) motifs [23-24]. Other motifs 25 commonly found among VAP-interacting proteins in different eukaryotes are the oxysterol26 binding motifs, and/or phosphoinositide-binding pleckstrin homology (PH) domains which are

27 putatively involved in the docking of VAPs to the apposed membranes [2, 24-25] (Figure 3B). In 28 Arabidopsis, the best characterized VAP is VAP27-1, which is anchored to the ER via a single C- 
1 terminal transmembrane (TM) domain. VAP27-1 attaches to the PM using a complex that

2 involves the actin binding protein NET3C, and the turnover of this complex is mediated by the

3 actin and microtubule networks [21]. In a recent study, nine additional Arabidopsis VAPs, divided

4 into three clades (VAP27-1 to -10), have been identified based on homology to the N-terminal

5 MSP domain of VAP27-1 [10]. Using different VAP27-GFP fusions it was shown that several VAPs

6 from clades I and III (containing TM domains) were localized to ER-PM contact sites, whereas

7 VAPs from clade II (lacking a TM domain) were PM-localized suggesting that the TM anchor might

8 be essential for VAP localization and/or tethering to the ER [10]. In developmental terms,

9 pleiotropic defects in pollen, seeds, and root hair development were observed in VAP27 RNAi

10 lines and in plants carrying VAP27-GFP fusions [10]. However, the individual contribution of

11 different VAP27 isoforms (and/or MCS at large), to those developmental processes is still largely

12 unknown.

13 In Arabidopsis, synaptotagmin (SYT) tethers are encoded by at least five genes (SYT1 -

14 SYT5), which are the closest homologs of the well-characterized mammalian extended 15 synaptotagmins and yeast tricalbin membrane tethers $[9,26]$. SYT1 is the best characterized

16 member of the family, and its role as an ER-to-PM tether has recently been established $[9,13]$.

17 SYT1 contains a N-terminal TM domain anchored to the ER membrane, a 40 amino acid linker

$18(\sim 15 \mathrm{~nm})$ that, as in yeast [23], might be of sufficient length to bridge the cytosolic gap, and a 19 cytoplasm-exposed synaptotagmin-like mitochondrial lipid binding protein (SMP) domain that in 20 mammals regulates the assembly of protein-protein complexes, and the establishment of inter21 organelle tubular paths for lipid transfer [27-29] (Figure 3A). In SYT1, docking to the PM is likely 22 established by two independently folded high-affinity $\mathrm{Ca}^{2+}$-dependent lipid-binding domains (C2 23 domains) which generate electrostatic interactions with negatively charged phospholipids [9, 30-

24 31] (Figure 3A). SYT1 has recently been shown to interact with elements of the exocytotic soluble $25 \mathrm{~N}$-ethylmaleimide-sensitive factor attachment protein receptor (SNARE) complex, involved in 26 vesicle fusion [11], the stigmasterol binding protein ROSY1 [32], and the plant reticulon proteins 27 RTNLB3 and RTNLB6 involved in ER tubulation and the establishment of ER membrane curvature 28 [33]. In developmental terms, SYT1 loss-of-function causes $\mathrm{Ca}^{2+}$-dependent susceptibility to 29 abiotic stresses that impose changes to the cell turgor (i.e. osmotic and freezing stresses) [30, 
$134]$, and it was proposed that SYT1-containing MCS act as deformable platforms that coordinate

2 PM responses to mechanical stress in a process likely involving the cortical cytoskeleton [9].

3 Interestingly, a recent study showed that SYT1 negatively regulates immune secretory pathways

4 during fungal infection [11] in a process that might be linked to the observed SYT1 enrichment in

5 the extrahaustorial membrane [35]. Whether the SYT1 enrichment is a plant response aimed at stabilizing the PM during fungal penetration, or a fungal strategy used to achieve efficient MCSmediated macromolecular exchange between the haustoria and the host remains unclear. No information is currently available about the other members of the SYT family in plants except conflicting results regarding Arabidopsis SYT2 subcellular localization and its putative roles in conventional and/or unconventional secretion[36-37].

Remarkably, the search for plant homologs of known MCS components also highlights 12 clear differences between plants and other eukaryotes regarding MCS component distribution 13 and function. An illustrative example of differential MCS components is observed at 14 mitochondrial contact sites. In most eukaryotes, the biogenesis of the mitochondrial membranes 15 requires non-vesicular phospholipid exchanges that likely occurs at inter-organelle MCSs [38-39]. 16 In yeast, mitochondrial lipid transfer is thought to be mediated by tethering complexes that form 17 the ER-mitochondria encounter structure (ERMES). However, the presence of functional 18 mitochondria in the absence of an ERMES refutes the idea of ERMES as the sole mediator of lipid 19 transfer to mitochondria [40-41]. Remarkably, ERMES tethers are not conserved in plants, 20 suggesting that the exchange of lipids required for the biogenesis of mitochondrial membranes 21 occurs through a different mechanism. In this context, a recent study postulates that a different 22 Endoplasmic Reticulum Membrane Protein tethering Complex (EMC) might be involved in 23 phospholipid transfer in different eukaryotes [41], including plants [42]. However, conclusions 24 that can be drawn about plant-specific MCS components and complex plant MCS functions from 25 homology searches alone are limited. For instance, the putative presence of an EMC complex in 26 plants does not preclude that alternative plant-specific MCS such as mitochondrial-plastid 27 contact sites (described below), might use different machineries, and/or involve plant-specific 28 lipid transfer mechanisms. In this context, the identification and characterization of tethering mutants in plants using genetic, cell biology, and molecular approaches is a valuable resource to 
1 identify novel MCS machineries and MCS functions that could then be extrapolated to other 2 eukaryotic systems.

\section{Unique Plant MCS architectures and functions}

\section{Plasmodesmata: ER-PM contact sites regulating inter-cellular communication}

One specialised type of plant MCS that is unique both amongst ER-PM contact sites and eukaryotic cell junctions are plasmodesmata (PD). PD are intercellular cytoplasmic channels that connect plant cells across the cell wall and constitute a major pathway for plant intercellular signalling and tissue patterning [43-45]. PD are structurally unique, with both the ER and the PM running through the pores, forming two membrane tubules concentrically arranged with an overall diameter of about $40 \mathrm{~nm}$ (Figure 2A, B). Membrane apposition at PD is unusually close

11 ( $10 \mathrm{~nm}$ distance) and the ER (termed desmotubule within PD) is linked to the PM on all sides

12 throughout the entire length of the channels by proteinaceous spoke-like elements [46], which 13 remain unidentified, although both SYT1 and VAP27-1 have recently been localised to PD [10, 14 13].

Although PD qualify as MCS [19], little is known about the function of ER-PM tethering at PD, and its possible contribution to controlling cell-to-cell connectivity remains speculative. PD may be engaged in the same processes that have been hypothesized for other types of ER-PM junctions, such as non-vesicular lipid transfer, or $\mathrm{Ca}^{2+}$ signalling [6-8], yet the unusual biophysical properties of PD (due to their highly curved membranes) together with their functional, lipidomic and proteomic specialization [33, 47-48] indicate that within the channels, communication

21 between ER and PM may act through mechanistically distinct pathways.

A recent characterisation of the Arabidopsis PD lipidome revealed a unique PD lipid profile

23 with enrichment in sterols and sphingolipids compared to the bulk PM [48]. This study concluded 24 that the generation of lipid micro-domains was essential for PD function and required the 25 delivery of specialized lipids and proteins to the PD membranes. How the differential distribution 26 of lipid species between the PD pore and the cellular PM occurs is currently unknown, but direct 27 trans-organelle ER-PM lipid transport, either within the channels or in their vicinity, may explain 
1 how PD could locally and rapidly regulate the movement of lipid species, and thereby the specification of their membranes.

In addition to putatively promoting inter-organelle molecule exchange, PD membrane tethering may also impact PD function by modulating their permeability. Current models propose that the PD size exclusion limit depends on the physical dimensions of the cytoplasmic compartment, which is itself a function of ER-PM spacing [19]. In this context, the $\beta$-1,3-glucan callose is viewed as the main regulator controlling the PD intermembrane gap [49-51]. Callose deposition or removal in the extracellular space around PD would dictate the size exclusion limit of the pores by pushing the PM against the desmotubule or releasing it, hence altering the physical dimensions of the cytoplasmic compartment. Numerous studies have indeed reported modification of PD size exclusion limit through callose, in response to a wide range of

12 developmental, biotic, and abiotic stimuli [19]. However, in addition to callose, PD membrane 13 tethering elements may also contribute to the regulation of PD permeability. Supporting this 14 possibility, elevated cytoplasmic $\mathrm{Ca}^{2+}$ in Arabidopsis causes a rapid and transient reduction in PD 15 permeability [52] that, as in mammals, might be mediated by the action of ER-PM protein tethers 16 [53-54]. In this scenario, the generation of $\mathrm{Ca}^{2+}$ spikes might activate PD-localized $\mathrm{Ca}^{2+}$-sensitive contractible tether proteins, such as SYTs, reducing the available space in the cytoplasmic compartment. Still, there are other possibilities, such as the release of ER $\mathrm{Ca}^{2+}$ stores mediated by PD-localized calreticulins [55], and/or the $\mathrm{Ca}^{2+}$-induced local biosynthesis of callose [51] that could explain the observed $\mathrm{Ca}^{2+}$-mediated PD closure. Eventually, a more comprehensive understanding of PD MCS function will come from the identification of PD membrane tethering elements, their interacting proteins and lipid targets, as well as a better access to the dynamics of their 3D ultrastructural arrangement.

An important aspect of PD permeability is that they are the only pathway available for cell-to-cell spread of plant viruses [56-57]. For this, viruses encode movement proteins which target and dilate PD, and mediate shuttling of viral genomes through the channels, past the PD

27 tethers. Viruses also interact with MCS at another stage of their infection: RNA viruses replicate 28 on modified host membranes, which are often heavily reorganised into complex 'virus factories', consisting of densely stacked, reticulated or invaginated lipid bilayers [58-59]. Membrane tethers 
1 involved in organising MCS might be co-opted for such 'virus factories'. Indeed, Tomato bushy 2 stunt virus, a plant RNA virus that is used as a model to study replication because it can infect

3 yeast cells, recruits yeast Scs2 and its plant homolog VAP27-3 (PVA12)[10], as well as yeast ORP1

4 and plant ORP3A lipid shuttling proteins to induce membrane proliferation at its replication site

$5 \quad[12,60]$. Intriguingly, movement- and replication-related interactions of plant viruses with MCS

6 might functionally overlap. There is now evidence for different viruses that establish replication

7 sites in modified membranes right at the entrances of PD $[13,61]$. It is not yet clear whether this

8 spatial coordination of movement and replication just facilitates speed and efficiency of virus

9 transport through PD, or plays a direct role in modifying and opening the channels, but the ER-

10 PM tether SYT1 is required for movement of a number of different viruses and recruited to PD

11 during infection [13, 62], favouring a more direct role of ER-PM contacts. Clearly, a more detailed

12 understanding of plant MCS will provide key clues to some fundamental unanswered questions

13 in plant intercellular communication and disease.

\section{Chloroplast-ER contact sites: lipid homeostatic modules in plants}

15 In addition to ensuring chemical energy production through photosynthesis, chloroplasts

16 are also required for the synthesis of amino acids, tocopherol, carotenoids, and fatty acids (FAs)

17 that are essential membrane lipid precursors. Since chloroplasts participate in core biosynthetic

18 pathways required for plant cell function, they establish continuous molecular exchanges with

19 their extra-plastidial environment. In the context of non-vesicular exchanges, early electron

20 microscopy studies reported the presence of regions of close membrane apposition between

21 chloroplast and ER membranes that might represent chloroplast-ER contact sites (also known as

22 plastid associated membranes; PLAMs) [63-64] More recent live imaging studies coupled with

23 laser scalpel and optical tweezers, quantified the strength of those putative membrane

24 attachment sites and established that under forces of up to $400 \mathrm{pN}$ the ER and the chloroplast

25 membranes remain physically attached [65].

The chloroplasts and ER membranes also have intertwined functional relationships as the

27 majority of chloroplast-synthesized FAs are exported to the ER to be assembled into storage and

28 membrane-forming glycerolipids. A fraction of these glycerolipids is then cycled back to the 29 chloroplast for the production of galactolipids, which are the predominant lipids of chloroplast 
membranes [14-15]. Although the mechanisms underlying such lipid transfer are not well understood, PLAMs are starting to emerge as sites of lipid exchange where the concentration of

3 lipid export machinery, and the close proximity of the membranes, might promote non-vesicular 4 lipid shuttling events [14-15, 66]. One example of such lipid transfer machinery associated with 5 PLAMs are members of the trigalactosyldiacylglycerol (TGD) protein family involved in the transfer of polar lipids from the ER to the chloroplast [67-69]. While TGD1,2,3 form an ABC 7 transporter complex and localise in the plastid inner envelope membrane, TGD4, a transmembrane lipid transfer protein, possesses a dual localisation being associated with both 9 the outer envelope and the ER membranes [68-69]. Recent work has identified the small glycinerich protein TGD5 as an additional partner that would bridge the TGD4 at the ER-chloroplast outer 11 membrane MCS to the TGD1-3 complex in the inner envelope [67]. The emerging picture is that

12 the TGD complex may provide a conduit to shuttle ER-derived lipids from the ER membrane 13 across the two chloroplast membranes for thylakoid lipid assembly [67]. In addition to promoting 14 molecular transfer via specific transporters, PLAMs were recently proposed to act as specialised 15 platforms for ER-plastid trans-acting enzymatic activity where ER-localized enzymes can directly 16 access chloroplast-located precursors and vice versa [20,70].Through an elegant trans-organellar complementation assay, it was shown that retargeting plastid-localized tocopherol and carotenoid enzymes to the ER was sufficient to achieve significant complementation of the mutated pathway activities in the plastid envelope [70]. These results implied that ER-retargeted enzymes could directly access the nonpolar metabolites in the plastid envelope, presumably 21 through PLAMs. As a consequence, a model whereby PLAMs could facilitate the establishment of 22 a stable hemi-fusion (apposed membranes forming a joined bilayer) enabling enzymes in either 23 organelle to access substrates from both compartments was proposed [20, 70] (Figure 2C).

24 Whether or not hemi-fusion-based trans-organellar complementation is indeed taking place at 25 ER-chloroplast contact sites, and how such a membrane arrangement could be formed and 26 stabilized by MCS components remains unknown.

Although the intriguing data described above support the existence of functional MCS between the ER and the chloroplasts, their molecular architecture has yet to be established. The emerging picture is that the structural and functional relationship between the ER and the 
1 chloroplast is complex, and multiple ER-Chloroplast contact sites are likely to co-exist. Moreover,

2 the ER may interact with specialised sub-domains of the chloroplast membranes as exemplified

3 by the ER contacts with plastid membrane extrusions called stromules.

\section{Stress-inducible contact sites: Stromules, peroxules and mitochondrial-plastid junctions}

Stromules are stroma-filled tubular protrusions that extend from the chloroplast outer surface and often associate with the nucleus, ER, and PM [71-72] (Figure 2A). These 0.35-1.5 $\mu \mathrm{m}$ diameter structures retract and extend constantly, enabling the movement of stromal and outer envelope components to the target organelles [73]. Stromule formation is dependent on intrinsic factors, such as plastid size and density [74], but they can also be formed in response to environmental conditions that modify the redox status of the chloroplast, such as bacterial and viral infections, high light intensity, and application of photosynthetic electron transport chain inhibitors $[16,73]$. These stress-induced chloroplast modifications are thought to be required for the establishment of stromules-to-nucleus contact sites enabling the direct translocation of chloroplast-localized proteins to nuclei [75]. Moreover, accumulating evidence shows that many nuclear proteins, including transcription factors, are sequestered in plastids and translocate to the nucleus in response to stress [76-78]. Examples of stress-mediated translocations at stromule-nuclear junctions includes the membrane-bound transcription factor PTM that is localized to the outer chloroplasts envelope and undergoes proteolytic cleavage at the $\mathrm{N}$ terminus and relocation to the nucleus under high light conditions [76]; the transcription factor WHIRLY1 that accumulates as oligomers at chloroplasts, but upon changes in the chloroplast redox-state dissociates into monomers that translocate to the nucleus [77]; and the rhodanese sulphurtransferase N RECEPTOR INTERACTING PROTEIN 1 (NRIP1) that translocates to the nucleus in the presence of the viral p50 helicase effector in a process likely occurring at stromulenuclear contact sites [78]. Although none of these examples conclusively demonstrate that the exchange of biophysical signals and cargoes took place at the point of contact between stromules and nucleus, they point towards the establishment of stress-inducible contact sites as a suitable model for direct communication between these organelles. We envision that an in depth analysis of these plant-specific structures will provide important insights into the molecular basis of inducible inter-organelle communication systems in eukaryotes. 
Changing the redox state of chloroplasts not only promotes the formation of stromules,

2 but also that of peroxules (extensions from peroxisomes, Figure 2A), which are involved in the

3 detoxification of reactive oxygen species [79]. Peroxules form in response to hydroxyl radical-

4 inducing conditions, such as high light, [80], follow the dynamics of ER tubules [17], and extend

5 over the surface of chloroplasts and mitochondria establishing peroxisome-organelle contact sites [17, 79-80]. Recently different methodologies have been used to analyze the interaction

7 between peroxisomes and chloroplasts. For example, femtosecond laser technology combined with confocal microscopy was used to analyze the adhesion between the two organelles and

9 demonstrated that high light intensities promote the establishment of peroxisome-chloroplastmitochondria three-way junctions, and induce a 3-fold increase in the amount of force needed

11 to disturb chloroplast-peroxisome interactions [81]. A different study combining optical tweezers

12 and TIRF microscopy identified peroxules as the tethering force between peroxisomes and 13 chloroplasts [82]. Although these studies represent important advances in the structural 14 elucidation of stromules and peroxules, we still lack a demonstration of direct metabolic 15 exchange between organelles through peroxules and stromules. This is technically not trivial 16 because both structures are highly sensitive to conventional fixation techniques and laser 17 exposure.

Finally, the formation of stress-inducible mitochondrial protrusions and mitochondrialplastid contact sites [83-84] suggests that mitochondrial MCS might have a role in the biogenesis and function of plant mitochondrial membranes. This role has been described for the plastidsynthesized lipid digalactosyldiacylglycerol (DGDG) that is kept within the plastid membranes in

22 normal conditions but is exported towards the mitochondrial membranes under phosphate 23 starvation [84]. Although the concrete mechanism of DGDG transfer to mitochondria is still 24 unknown, a recent study points towards a large mitochondrial transmembrane lipoprotein 25 complex $(\mathrm{MTL})$, containing a homolog of the yeast mitochondrial inner membrane component 26 Mic60, as an important regulator of plastid mitochondria contact site establishment during 27 phosphate starvation [39]. Together these results suggest that evolutionarily conserved 28 mitochondrial MCS components, such as Mic60, might have undergone processes of sub- and/or neo-functionalization to adapt to the unique plant cell environment. 


\section{Concluding remarks and future perspectives}

Although plant MCS have been morphologically described for over 40 years, we are just starting to dissect their biochemical, biophysical and functional characteristics. Recent advances

4 in imaging techniques such as TEM tomography (Figure 1B-C) and fluorescent live-cell imaging (Figure 1E-G) are starting to unravel the intricate spatial and dynamic organization of these plant membrane structures. In parallel, the genetic identification of plant MCS machineries and the functional characterization of plant MCS architectures is providing mechanistic insights into the molecular events involved in the docking of closely apposed membranes, the non-uniform distribution of lipids between MCS membranes, and the regulation of inter-organelle and intercellular communication. Together, these results show plant MCS as essential communication

11 nodes required for the coordination of multiple metabolic activities. Still, many questions regarding MCS molecular composition and their impact on the physiology and dynamics of most organelles remain unanswered (see Outstanding Questions). We envision that upcoming plant MCS research will be focused on establishing commonalities between plant MCS and other eukaryotic MCS regarding conserved functions, such as the establishment of $\mathrm{Ca}^{+2}$ release mechanisms between the ER and the extracellular space, or non-vesicular lipid transfer processes whose presence in plants has been inferred from other eukaryotic organisms. In this context, the identification of additional plant MCS components common to all eukaryotes will enable an indepth assessment of these functions in plants. In parallel, the identification of plant-specific MCS tethering complexes and MCS components will uncover unique plant MCS functions not previously inferred from other eukaryotes. For this aim, the functional and structural analysis of plant-specific MCS architectures such as PD and stress-inducible MCS are two promising research avenues. Finally, plant MCS research will analyze the putative roles of different plant MCS in specific processes such as the transfer of lipidic cell wall components, or the release of vacuolar contents to the apoplast, where a contribution from non-vesicular transport mechanisms has been proposed, but not established, and assess the relative importance of MCS-mediated

27 compared to vesicular transport. Together, these areas of plant MCS research will uncover the 


\section{1 fitness and development, and will also provide new insights into MCS of other eukaryotic}

2 organisms.

4 BOX 1. How "tight" should a MCS junction be? Morphological criteria for ER-PM contact sites.

5 Structurally, plant ER-PM contact sites are substructures of, and continuous with, the cortical ER

6 (CER). TEM microscopy (Figure 1A), and electron tomography of plant cells shows that the cER forms an

7 extensive network that can be either micrometres away from, or form contact sites with the PM (Figure

8 1B-1C, Supplemental Video 1). This continuity between ER-PM contact sites and cER makes it challenging

9 to define how closely apposed the two membranes must be to constitute a functional MCS, especially

10 using confocal microscopy where the entire cortical cytoplasm can be contained within just a few optical

11 sections. Freeze-fracture studies showed contact sites with an intermembrane distance of $<20 \mathrm{~nm}$

12 between the CER and PM, and these were often situated at sites where secretory vesicles had recently

13 fused [64]. These dimensions are congruent with the view that an important functional hallmark of ER-

14 PM contact sites is focusing trafficking and/or metabolism, thus the size of the gap between the ER and

15 PM bilayers should reflect the dimensions of ER-anchored enzymes acting on substrates in the PM. In

16 yeast, functional assays have demonstrated that for protein-protein interactions to occur across ER-PM

17 contacts, the ER must be within $20 \mathrm{~nm}$ of the PM [23]. Based on these studies and the early reports from

18 electron microscopy [64, 85], the morphological criteria for plant ER-PM contact sites can be defined as a

19 region where the two membranes are $\leq 20 \mathrm{~nm}$ apart with ribosomal exclusion.

BOX 2. The complex interplay between cytoskeleton and ER-PM contact sites in plants.

Plant ER-PM contact sites exist in a cortical cytoplasm that is unique compared to yeast or

22 mammalian cells, because the large central vacuole fills most of the typical plant cell, so that the cell

23 cortex is sandwiched into a thin layer between the vacuole and PM (Figure 1B-1C). A prominent feature

24 of the cortical cytoplasm is the interphase cytoskeleton array consisting of dynamic microtubules lining

25 the PM (Figure 1D). There are fine actin microfilaments, Golgi moving via myosin motors on actin filament

26 bundles, and constant secretory and endocytic vesicle traffic to accommodate plant cell wall growth. Since

27 microtubules and microfilaments are enriched in the plant cortical cytoplasm, some dynamic interplay is

28 predicted between the cytoskeleton and ER-PM contact sites. The acto-myosin system is important for

29 remodelling and mobilizing the bulk of the cER [85-86], and in vitro, ER tubules can move on filamentous

30 actin in the presence of myosins and cofactors [87]. In contrast to the dynamic cER, ER at contact sites is

31 characterized by a static nature, which has been demonstrated by their persistence in live-cell mapping 
1 analysis of ER movement [21, 86]. ER-PM contact sites are not sensitive to disruption by actin

2 microfilament disrupting agents such as Latrunculin B [9-10, 21], although NET3C is an actin binding

3 protein and the turnover of NET3C within contact sites is affected by loss of microfilaments [21]. The

4 interplay between microtubules and cortical ER in plants is more subtle. While ER dynamics are dominated

5 by the actin cytoskeleton, cortical microtubules play roles in maintaining the fine cER tubule network

6 through slow ER tubule extension and ER anchoring [88]. As the gap between the bilayers at ER-PM

7 contact sites is less than the $25 \mathrm{~nm}$ diameter of microtubule, it makes sense that contact sites and

8 microtubules are mutually exclusive immediately adjacent to the PM. The localization of ER-PM contact

9 site components such as SYT1 and VAP27/NET3C clearly support this [9-10], as do TEM data (e.g. Figure 10 1D).

\section{BOX 3. Visualizing MCS: Context and Dynamics}

12 Since eukaryotic MCS are nanoscale structures, detailed characterization is based on transmission

13 electron microscopy (TEM) data (Examples in Figure 1A-D and [64, 85, 89-90]). TEM sample preparation

14 methods can have a dramatic impact on the preservation of the plant cortical ER and PM, as the PM is

15 particularly sensitive to osmotic perturbation during chemical fixation due to the fact that it is under

16 turgor pressure [91]. In chemically fixed yeast cells, TEM revealed over 1000 ER-PM contact sites of $30 \mathrm{~nm}$

17 or less per cell, while in high pressure frozen/freeze substituted yeast cells, where the native structure is

18 more accurately preserved, 10 sites per cell were observed [89]. These results suggest that cryo-fixation

19 provides a more reliable structural view of these specialized inter-organelle junctions. While TEM provides

20 high resolution information and cellular context in well-preserved cells, it can only provide information

21 on static proximity and the presence of putative MCS components [9, 21]. Advances in light microscopy,

22 such as optical tweezers, have provided methods to directly demonstrate the interactions between

23 organelles, such as ER and plastid [65]; ER and Golgi [92], and peroxisomes and plastids [82], as well as to

24 analyse their dynamic characteristics.

25

26

27

28

29

30 
2 Figure 1. Visualization of ER-PM contact sites using TEM and fluorescence live-cell imaging. A) TEM showing the CER (blue), the PM (brown), and a putative ER-PM contact site, in Arabidopsis roots. The arrow marks the position of a PD. B) TEM images of a cell in poplar shows the mutual exclusion between the microtubules ( $m t$, red) and the cortical ER (cER, blue) in regions adjacent to the plasma membrane (PM).(C-D) TEM tomography model of ER-PM contact sites in Arabidopsis roots illustrates the complexity of the CER-PM interface. TEM tomography integrates series of 2D TEM images (single image in C) and model them into detailed 3D reconstructions (D). Plasma membrane is dark red, tonoplast is orange, internal ER surfaces are green, external ER surfaces are blue and vesicles are pink. (E to G) Confocal microscopy visualization of putative ERPM contact sites architectures labelled by the SYT1proSYT1-GFP marker [9] in different Arabidopsis cell types. E) Reticulated ER-PM contact sites labelled by the SYT1proSYT1-GFP marker in fully expanded leaf epidermal cells. F) Homogeneous distribution of cER-PM contact sites in a fully developed trichome. G) Different size ER-PM contact sites puncta observed in root epidermal cells.

Figure 2. (Key Figure) Specialized membrane contact sites in plants. (A) Schematic illustration of a plant cell, highlighting the formation of representative MCS between the endoplasmic reticulum (ER) and the plasma membrane (PM) at plasmodesmata (PD) inter-cellular channels (see also panel B); the ER and the chloroplast outer envelope (see also panel C); the ER/nuclear envelope and the chloroplast-derived stromule; the chloroplast and the peroxule (model is not to scale). (B) Close up view of ER-PM junction at PD showing the desmotubular ER extending through the cell wall and being linked on all sides to the PM by unidentified tethering elements. The gap between the two membranes maintains cytoplasmic continuity between plant cells and provides a path for molecules to traffic from cell-to-cell. The tethering elements of PD channels have the potential to regulate intercellular communication by either modulating the dimensions of the cytoplasmic compartment, and/or promoting exchange of molecules, such as lipids, between the two membranes. (C) Hypothetical hemifusion event between the ER and the chloroplast outer envelope membrane. Membrane hemifusion would account for direct access of enzymes from one membrane compartment to the non-polar substrates located on the juxtaposed membrane surface $[20,70]$.

Figure 3. Schematic representation of the functional domains of established and potential MCS tethers identified in the Arabidopsis genome. A) Putative Transmembrane tethers containing lipid transfer and lipid binding domains, B) Putative cytosolic tethers containing lipid transfer and lipid binding domains. Green oval, transmembrane domain; purple box, major sperm domain (MSP); blue box, pleckstrin homology (PH) domain; red box, synaptotagmin-like mitochondrial lipid binding protein (SMP) domain; yellow box, $\mathrm{Ca}^{2+}$-dependent lipid-binding domain (C2 domain); orange box: Oxysterol binding protein (OBP) domain; green box, steroidogenic acute regulatory-related lipid transfer protein domain (StART). NTMC: $\mathrm{N}$-terminal-transmembrane-C2 domain proteins. The VAP27 clade separation is derived from [10], and the ORP types classification from [95]. Each Arabidopsis tether is identified by its gene model locus. Additional putative MCS components have been identified in recent studies. [11, 32-33]. 


\section{Acknowledgements}

We apologize to any authors whose relevant work on MCS have not been cited

3 inadvertently or owing to length constraints. We would like to thank Cyril Dejean from SciLight

4 (http://www.scilight.eu) for preparing illustrations for the review. This work was supported by

5 the Canada Research Chairs Program (to AR), two Natural Sciences and Engineering Research

6 Council of Canada (NSERC) Discovery Grants (to AR and LS), a Research Training Fellowship from

7 the Ministerio de Economía y Competitividad (FPI-BES 2012-052324 to JP-S), the Ministerio de

8 Ciencia e Innovación (co-financed by the European Regional Development Fund; grant

9 no. BIO2014-55380-R to MAB), the UK Biotechnology and Biological Sciences Research Council

10 (BBSRC grant BB/M007200/1 to JT), and the French National Agency for Research (Grant ANR-

11 14-CE19-0006-01 to EB). Thanks to the UBC Bioimaging facility for assistance with electron

12 tomography, as well as to Laura Vanbezouwen and Heather McFarlane for modeling, and

13 Eunkyoung Lee for generating the confocal microscopy images. 


\section{References}

2 1. Lunn, J.E. (2007) Compartmentation in plant metabolism J. Exp. Bot. 58, 35-47.

3 2. Prinz, W.A. (2014) Bridging the gap: membrane contact sites in signaling, metabolism, and organelle 4 dynamics. J. Cell Biol. 205, 759-769.

3. Levine, T.P and Patel, S. (2016) Signalling at membrane contact sites: two membranes come together to handle second messengers. Curr. Opin. Cell .Biol. 39, 77-83.

4. Helle, S.C.J. et al. (2013) Organization and function of membrane contact sites. Biochim. Biophys. Acta - Mol. Cell Res. 1833, 2526-2541.

5. Phillips, M.J. and Voeltz, G.K. (2016) Structure and function of ER membrane contact sites with other organelles. Nat. Rev. Mol. Cell Biol. 17, 69-82.

6. Dickson E.J. et al. (2016) Regulation of calcium and phosphoinositides at endoplasmic reticulummembrane junctions. Biochem. Soc. Trans. 44, 467-473.

7. Drin, G. et al. (2016) New molecular mechanisms of inter-organelle lipid transport._Biochem. Soc. Trans. 44, 486-492.

8. Henne, W.M. et al. (2015) Molecular mechanisms of inter-organelle er-pm contact sites. Curr. Opin. Cell Biol. 35, 123-130.

9. Pérez-Sancho J. et al. (2015) The Arabidopsis synaptotagmin1 is enriched in endoplasmic reticulumplasma membrane contact sites and confers cellular resistance to mechanical stresses. Plant Physiol. 168, 132-143.

10. Wang, P. et al. (2016) Plant VAP27 proteins: domain characterization, intracellular localization and role in plant development. New Phytol. 210, 1311-1326.

11. Kim, H. et al. (2016) Synaptotagmin 1 negatively controls the two distinct immune secretory pathways to powdery mildew fungi in Arabidopsis. Plant Cell Physiol. doi: 10.1093/pcp/pcw061

12. Barajas, D. et al. (2014) Co-opted oxysterol-binding ORP and VAP proteins channel sterols to RNA virus replication sites via membrane contact sites. PLoS Pathog. 10, e1004388.

13. Levy, A. et al. (2015) Synaptotagmin SYTA Forms ER-Plasma Membrane Junctions that Are Recruited to Plasmodesmata for Plant Virus Movement. Curr. Biol. 25, 2018-2025.

28 14. Hurlock, A.K. et al. (2014) Lipid trafficking in plant cells. Traffic 15, 915-932.

29 15. Block, M.A. and Jouhet, J. (2015) Lipid trafficking at endoplasmic reticulum-chloroplast membrane contact sites. Curr. Opin. Cell Biol. 35, 21-29

16. Brunkard, J.O. et al. (2015) Chloroplasts extend stromules independently and in response to internal redox signals. Proc. Nat. Acad. Sci. USA. 112, 10044-10049. 
17. Sinclair, A.M et al. (2009) Peroxule extension over ER-defined paths constitutes a rapid subcellular response to hydroxyl stress. Plant J. 59, 231-242.

18. Jouhet, J. et al. (2004) Phosphate deprivation induces transfer of DGDG galactolipid from chloroplast to mitochondria. J. Cell Biol. 167, 863-874.

19. Tilsner J. et al. (2016) Staying Tight: Plasmodesmal Membrane Contact Sites and the Control of Cellto-Cell Connectivity in Plants. Annu. Rev. Plant Biol. 67, 23.1-23.28.

20. Mehrshahi, P. et al. (2014) Redefining the metabolic continuity of chloroplasts and ER. Trends Plant Sci. 19, 501-507.

21. Wang P. et al. (2014) The plant cytoskeleton, NET3C, and VAP27 mediate the link between the plasma membrane and endoplasmic reticulum. Curr. Biol. 24, 1397-1405.

22. Laurent, F. et al. (2000) Molecular cloning and partial characterization of a plant VAP33 homologue with a major sperm protein domain. Biochem. Biophys. Res. Commun. 270, 286-292.

23. Gatta, A.T. et al. (2015) A new family of StART domain proteins at membrane contact sites has a role in ER-PM sterol transport. Elife. doi: 10.7554/eLife.07253.

24. Murphy, S.E. and Levine, T.P. (2016) VAP, a Versatile Access Point for the Endoplasmic Reticulum: Review and analysis of FFAT-like motifs in the VAPome. Biochim. Biophys. Acta. doi: 10.1016/j.bbalip.2016.02.009

25. Saravanan. R.S. et al. (2009) The targeting of the oxysterol-binding protein ORP3a to the endoplasmic reticulum relies on the plant VAP33 homolog PVA12. Plant J. 58, 817-830.

26. Craxton, M. (2007) Evolutionary genomics of plant genes encoding N-terminal-TM-C2 domain proteins and the similar FAM62 genes and synaptotagmin genes of metazoans. BMC Genomics. 8:25

27. Saheki, Y. et al. (2016) Control of plasma membrane lipid homeostasis by the extended synaptotagmins.Nat. Cell Biol. 18, 504-515.

28. AhYoung, A.P. et al. (2015) Conserved SMP domains of the ERMES complex bind phospholipids and mediate tether assembly. Proc. Nat. Acad. Sci. USA. 112, E3179-E3188.

29. Schauder CM. et al. (2014) Structure of a lipid-bound extended synaptotagmin indicates a role in lipid transfer. Nature. 510, 552-555.

30. Schapire, A.L. et al. (2008) Arabidopsis synaptotagmin 1 is required for the maintenance of plasma membrane integrity and cell viability. Plant Cell. 20, 3374-3388.

31. Pérez-Sancho, J. et al. (2016) Analysis of Protein-Lipid Interactions Using Purified C2 Domains. Methods Mol. Biol. 1363, 175-187.

32. Dalal, J. et al. (2016) ROSY1, a novel regulator of gravitropic response is a stigmasterol binding protein, J. Plant Physiol. 196-197, 28-40. 
33. Kriechbaumer, V. et al. (2015) Reticulomics: protein-protein interaction studies with two plasmodesmata-localized reticulon family proteins identifybinding partners enriched at plasmodesmata, endoplasmic reticulum, and the plasma membrane. Plant Physiol. 169, 1933-1945.

34. Yamazaki, T. et al. (2008) Calcium-Dependent Freezing Tolerance in Arabidopsis Involves Membrane Resealing via Synaptotagmin SYT1. Plant Cell. 20, 3389-3404.

35. Bozkurt, T.O. et al. (2014) The Plant Membrane-Associated REMORIN1.3 Accumulates in Discrete Perihaustorial Domains and Enhances Susceptibility to Phytophthora infestans. Plant Physiol. 165, 1005-1018.

36. Zhang, H. et al. (2011) Golgi apparatus-localized synaptotagmin 2 is required for unconventional secretion in Arabidopsis. PLoS One doi: 10.1371/journal.pone.0026477.

37. Wang, H. et al. (2015) Arabidopsis Synaptotagmin 2 Participates in Pollen Germination and Tube Growth and Is Delivered to Plasma Membrane via Conventional Secretion. Mol. Plant. 8, 1737-1750.

38. Galmes, R. et al. (2016) ORP5/ORP8 localize to endoplasmic reticulum-mitochondria contacts and are involved in mitochondrial function. EMBO Rep. pii: e201541108.

39. Michaud, M. et al. (2016) AtMic60 Is Involved in Plant Mitochondria Lipid Trafficking and Is Part of a Large Complex. Curr. Biol. 26, 627-639.

40. Lang, A. et al. (2015) ER-mitochondria contact sites in yeast: beyond the myths of ERMES. Curr. Opin. Cell Biol. 35, 7-12.

41. Lahiri, S. et al. (2014) A conserved endoplasmic reticulum membrane protein complex (EMC) facilitates phospholipid transfer from the ER to mitochondria. PLoS Biol. 12(10):e1001969.

42. Wideman, J.G. (2015) The ubiquitous and ancient ER membrane protein complex (EMC): tether or not? F1000Res. 4: 624.

43. Bayer, E.M. et al. (2014) Specialised membrane domains of plasmodesmata, plant intercellular nanopores. Front. Plant Sci. 5, 507.44. Brunkard, J. O. et al. (2015) The cytosol must flow: intercellular transport through plasmodesmata. Curr. Opin. Cell Biol. 35, 13-20.

44. Brunkard, J. O et al. (2015) The cytosol must flow: intercellular transport through plasmodesmata. Curr. Opin. Cell Biol. 35, 13-20.

45. Otero S. et al. (2016) Symplastic communication in organ formation and tissue patterning. Curr. Opin. Plant Biol. 29, 21-28.

46. Ding B. et al. (1992) Substructure of freeze-substituted plasmodesmata. Protoplasma. 169, 28-41.

47. Fernandez-Calvino, L. et al. (2011) Arabidopsis Plasmodesmal Proteome. PLoS One. 6: e18880.

48. Grison M. et al. (2015) Specific membrane lipid composition is important for plasmodesmata function in Arabidopsis. Plant Cell 27, 1228-1250. 
49. Vatén A. et al. (2011) Callose Biosynthesis Regulates Symplastic Trafficking during Root Development. Dev. Cell 21, 1144-1155.

3 50. Levy, A. et al. (2007) A plasmodesmata-associated beta-1,3-glucanase in Arabidopsis. Plant J. 49, 669682.

51. De Storme, N and Geelen, D. (2014) Callose homeostasis at plasmodesmata: molecular regulators and developmental relevance. Front. Plant Sci. 5:138.

52. Holdaway-Clarke, T.L. et al. (2000) Physiological elevations in cytoplasmic free calcium by cold or ion

8 injection result in transient closure of higher plant plasmodesmata. Planta. 210, 329-335

53. Chang, C.L. et al. (2013) Feedback regulation of receptor-induced Ca2+ signaling mediated by E-Syt1 and Nir2 at endoplasmic reticulum-plasma membrane junctions. Cell Rep. 5, 813-825.

54. Fernández-Busnadiego, R. et al. (2015) Three-dimensional architecture of extended synaptotagminmediated endoplasmic reticulum-plasma membrane contact sites. Proc. Natl. Acad. Sci. USA 112, E2004-13

55. Chen, M-H. et al. (2005) Effects of calreticulin on viral cell-to-cell movement. Plant Physiol. 138, 18661876.

56. Heinlein, M. (2015) Plasmodesmata: Channels for Viruses on the Move. In Plasmodesmata (1217 edn) (Heinlein,M., ed), pp. 25-52, Springer New York

57. Tilsner, J. et al. (2014) Plant Virus Movement.eLS, John Wiley \& Sons, Ltd, Chichester http://dx.doi.org/10.1002/9780470015902.a0020711.pub2

58. Cao, X. et al. (2015) Morphogenesis of Endoplasmic Reticulum Membrane-Invaginated Vesicles during Beet Black Scorch Virus Infection: Role of Auxiliary Replication Protein and New Implications of Three-Dimensional Architecture. J. Virol. 89, 6184-6195.

59. Xu, K. and Nagy, P.D. (2014) Expanding use of multi-origin subcellular membranes by positive-strand RNA viruses during replication. Curr. Opin. Virol. 9, 119-126.

60. Barajas, D. et al. (2014) Tombusviruses upregulate phospholipid biosynthesis via interaction between p33 replication protein and yeast lipid sensor proteins during virus replication in yeast. Virology. $471,72-80$.

61. Tilsner, J. et al. (2013) Replication and trafficking of a plant virus are coupled at the entrances of plasmodesmata. J. Cell Biol. 201, 981-995.

62. Uchiyama, A. et al. (2014) The Arabidopsis synaptotagmin SYTA regulates the cell-to-cell movement 
64. Staehelin, L.A. (1997) The plant ER: a dynamic organelle composed of a large number of discrete functional domains. Plant J. 11, 1151-1165.

65. Andersson, M.X. et al. (2007) Optical manipulation reveals strong attracting forces at membrane contact sites between endoplasmic reticulum and chloroplasts. J. Biol. Chem. 282, 1170-1174.

66. Bobik, K. and Burch-Smith, T.M. (2015) Chloroplast signaling within, between and beyond cells. Front Plant Sci. 6:781.

67. Fan, J. et al. (2015) Arabidopsis TRIGALACTOSYLDIACYLGLYCEROL5 Interacts with TGD1,TGD2, and TGD4 to Facilitate Lipid Transfer from the Endoplasmic Reticulum to Plastids. Plant Cell 27, 29412955.

68. Xu, C. et al. (2010) Lipid transport mediated by Arabidopsis TGD proteins is unidirectional from the endoplasmic reticulum to the plastid. Plant Cell Physiol. 51, 1019-1028.

69. Wang, Z. et al. (2012) TGD4 involved in endoplasmic reticulum-to-chloroplast lipid trafficking is a phosphatidic acid binding protein. Plant J. 70, 614-623.

70. Mehrshahi, P. et al. (2013) Transorganellar complementation redefines the biochemical continuity of endoplasmic reticulum and chloroplasts. Proc. Natl. Acad. Sci. USA 110, 12126-12131

71. Kwok, E.Y. and Hanson, M.R. (2004) Plastids and stromules interact with the nucleus and cell membrane in vascular plants. Plant Cell Rep. 23, 188-195.

72. Schattat, M. et al. (2011) Plastid Stromule Branching Coincides with Contiguous Endoplasmic Reticulum Dynamics. Plant Physiol. 155, 1667-1677.

73. Kumar, A. et al. (2014) Stromules. Adv. Plant Biol. 5, 189-20

74. Waters, M. T., Fray, R. G. and Pyke, K. A. (2004), Stromule formation is dependent upon plastid size, plastid differentiation status and the density of plastids within the cell. Plant J. 39, 655-667.

75. Caplan, J.L. et al. (2015) Chloroplast Stromules Function during Innate Immunity. Dev Cell. 34, 45-57.

76. Sun, X., et al (2011). A chloroplast envelope-bound PHD transcription factor mediates chloroplast signals to the nucleus. Nature Comm. 2: 477

77. Foyer, C.H., et al (2014). The functions of WHIRLY1 and REDOX- RESPONSIVE TRANSCRIPTION FACTOR 1 in cross tolerance responses in plants: a hypothesis. Phil. Trans. R. Soc. B. 369: 20130226.

78. Caplan, J.L. et al. (2008) Chloroplastic Protein NRIP1 Mediates Innate Immune Receptor Recognition of a Viral Effector. Cell. 132, 449-462.

79. Shai, N. et al. (2015) No peroxisome is an island - Peroxisome contact sites. Biochim. Biophys. Acta. DOI: 10.1016/j.bbamcr.2015.09.016.

80. Jaipargas, E.A. et al. (2016) High Light Intensity Leads to Increased Peroxule-Mitochondria Interactions in Plants. Front. Cell Dev. Biol. 4:6. doi: 10.3389/fcell.2016.00006. 
81. Oikawa, K. et al. (2015) Physical interaction between peroxisomes and chloroplasts elucidated by in situ laser analysis. Nature Plants. DOI: 10.1038/nplants.2015.57

3 82. Gao, H. et al. (2016) In Vivo Quantification of Peroxisome Tethering to Chloroplasts in Tobacco Epidermal Cells Using Optical Tweezers. Plant Phys. 170, 263-272.

83. Yamashita, A. et al. (2016) Formation of Mitochondrial Outer Membrane Derived Protrusions and Vesicles in Arabidopsis thaliana. PLoS One. 11(1):e0146717.

84. Jouhet, J. et al. (2004) Phosphate deprivation induces transfer of DGDG galactolipid from chloroplast to mitochondria J. Cell Biol. 167, 863-874.

9 85. Hepler, P. et al. (1990) Cortical endoplasmic reticulum in plants. J. Cell Sci. 96, 355-373.

86. Sparkes, I. et al. (2009) Movement and remodeling of the endoplasmic reticulum in nondividing cells of tobacco leaves. Plant Cell. 21, 3937-3949.

87. Yokota, E. et al. (2011) Myosin XI-dependent formation of tubular structures from endoplasmic reticulum isolated from tobacco cultured BY-2 cells. Plant Physiol. 156, 129-143.

14 88. Hamada, T. et al. (2014) Microtubules contribute to tubule elongation and anchoring of endoplasmic reticulum, resulting in high network complexity in Arabidopsis. Plant Physiol. 166, 1869-1876.

89. Perktold, A. et al. (2007) Organelle association visualized by three-dimensional ultrastructural imaging of the yeast cell. FEMS Yeast Res. 7, 629-638.

90. West, M. et al. (2011) A 3D analysis of yeast ER structure reveals how ER domains are organized by membrane curvature. J. Cell Biol. 193, 333-346.

91. Kiss, J.Z. et al. (1990) Comparison Of The Ultrastructure Of Conventionally Fixed And High-Pressure Frozen Freeze Substituted Root-Tips Of Nicotiana And Arabidopsis. Protoplasma. 157, 64-74.

94. Sparkes, I.A. et al. (2009) Grab a golgi: Laser trapping of golgi bodies reveals in vivo interactions with the endoplasmic reticulum. Traffic 10, 567-571.

95. Umate, P. (2011) Oxysterol binding proteins (OSBPs) and their encoding genes in Arabidopsis and rice. Steroids. 76, 524-529. 


\section{Outstanding Questions}

- Are MCS involved in other physiological processes that involve close membrane appositions such as organelle fission and fusions?

- Which are the tethering complexes involved in the establishment of different plant MCS and in particular, MCS that are unique to plants?

- Are there plant-specific tethering protein families not found in other eukaryotes?

- Are plant tethering protein families functionally divergent and/or have alternative functions to those described in yeast and mammals?

- Do the tethering complexes determine the functional specificity of the different MCS types?

- In each organelle, what is the relative importance of the non-vesicular lipid transport at plant MCS compared to vesicular lipid transport through the endomembrane system?

- Do plant ER-PM contact sites have $\mathrm{Ca}^{2+}$ release mechanisms similar to those observed in animal muscle cells?

- Apart from lipids and $\mathrm{Ca}^{2+}$ are there other molecules, such as lipidic cell wall components, that might be preferentially exchanged at MCSs?

- How do plant MCS sense environmental cues and trigger specific cellular responses?

- Which is the role of the plant cytoskeleton in the regulation of the MCS remodeling and plasticity? 

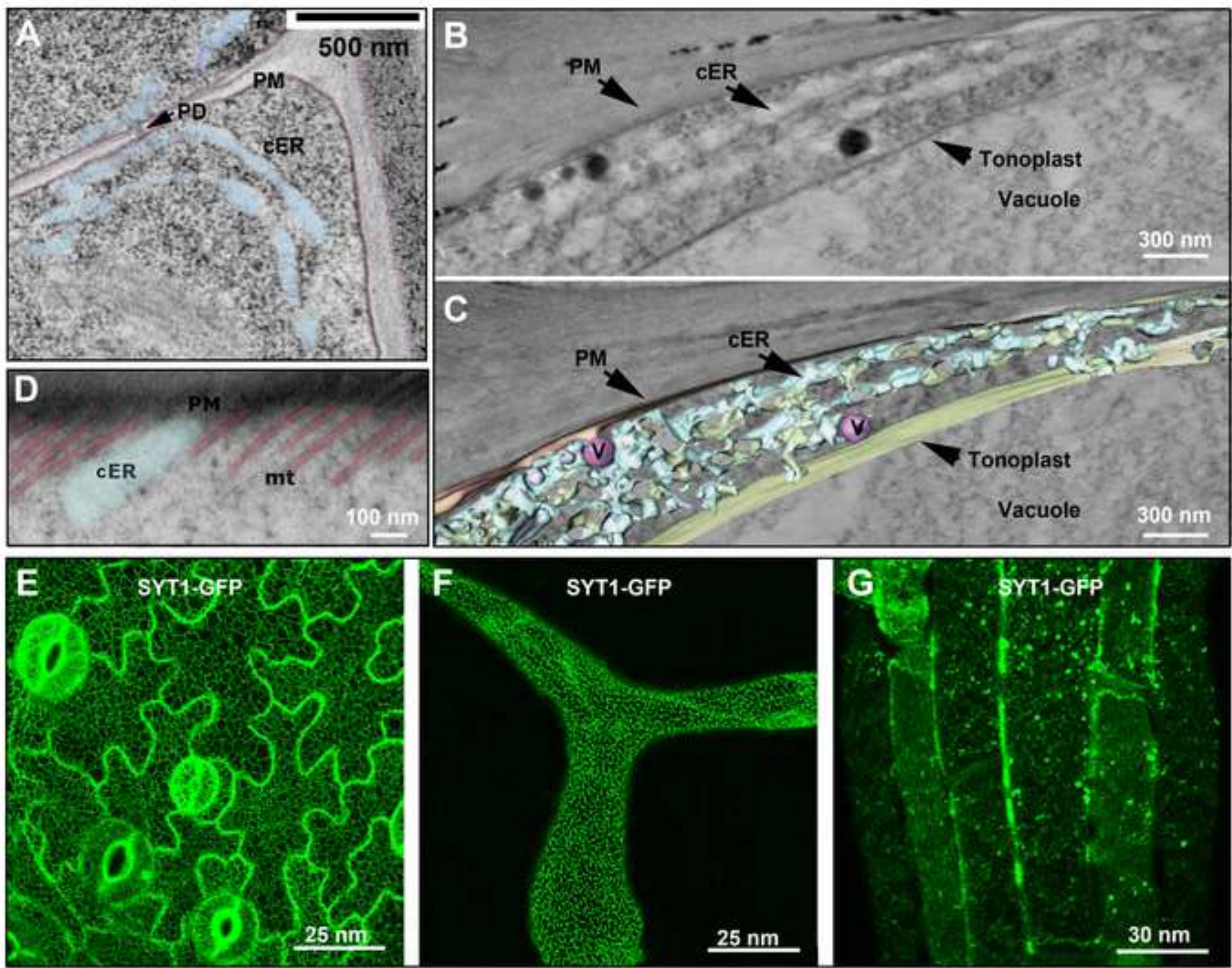

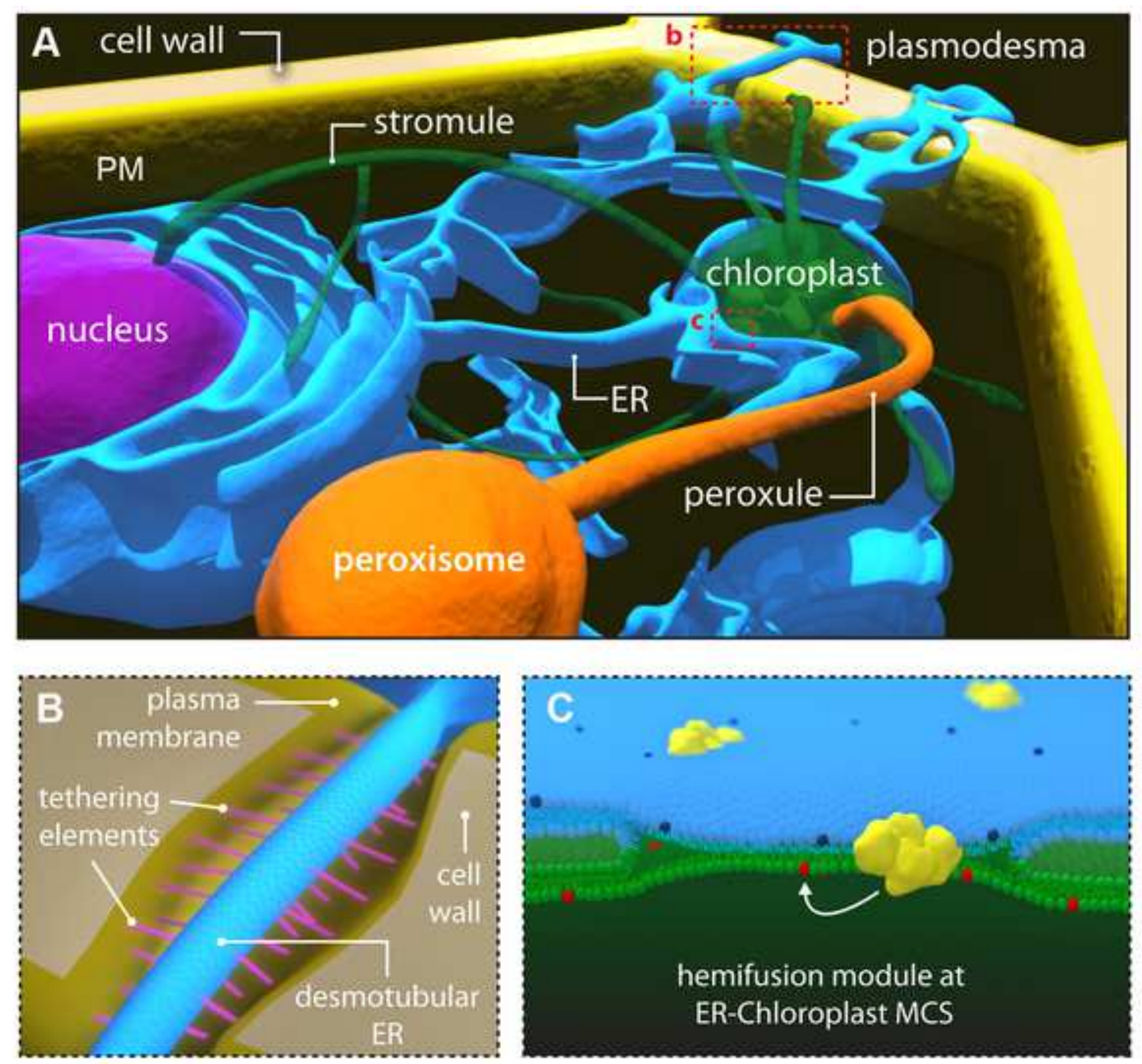
A

Putative Transmembrane tethers

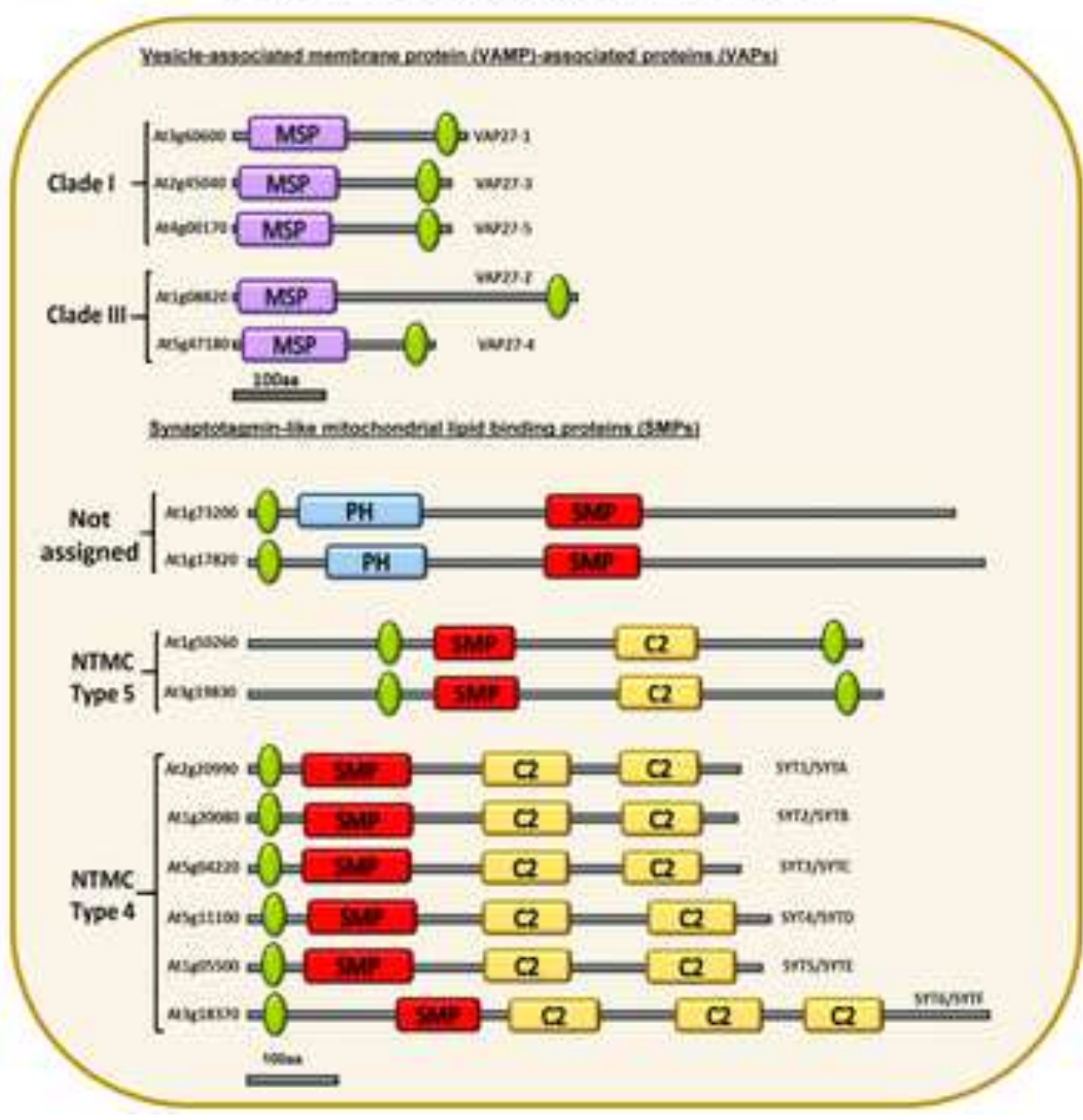

B

\section{Putative cytosolic tethers}

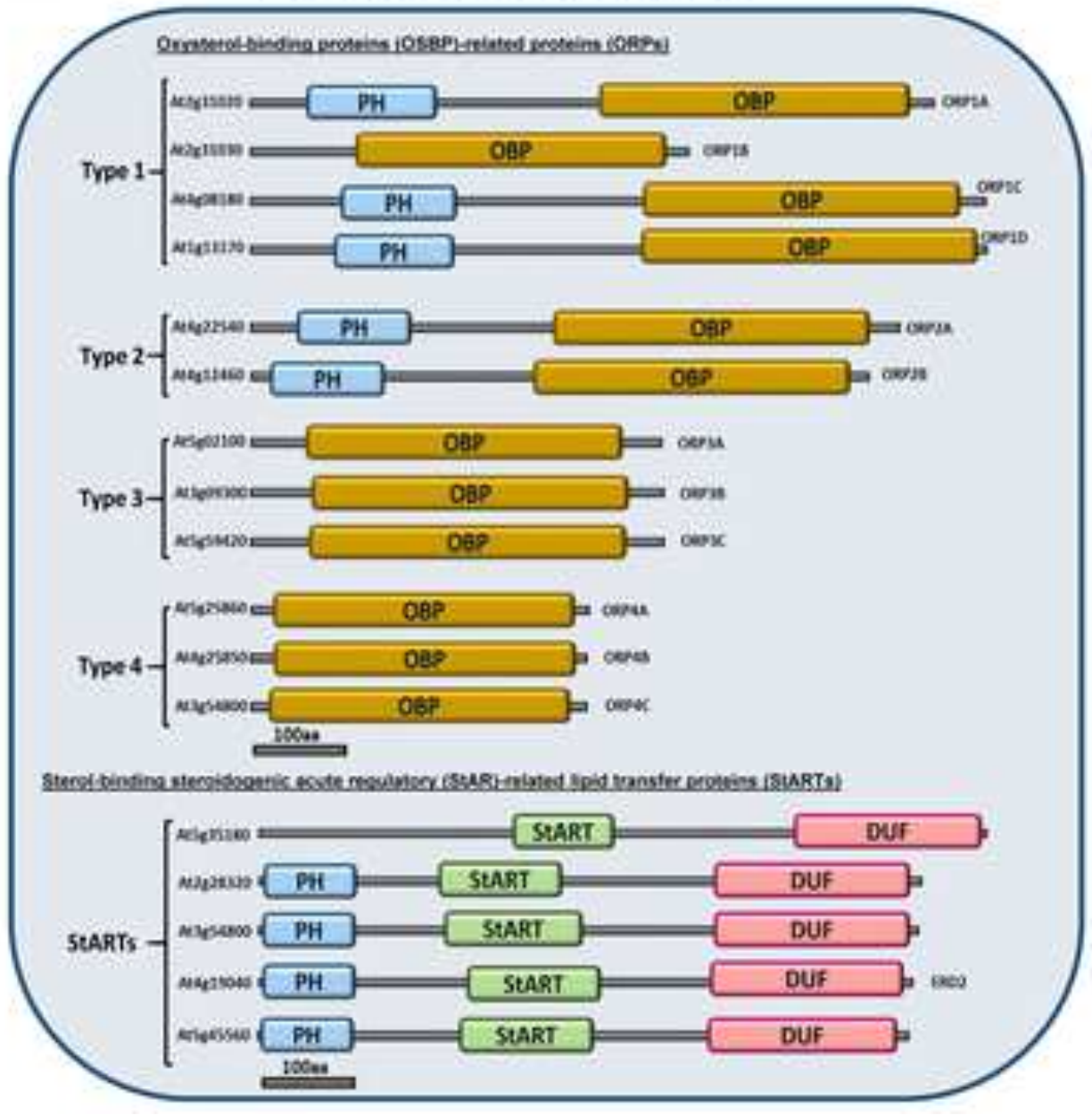


Click here to access/download Video Movie 1.wmv

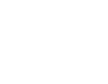


Click here to access/download

\section{Proposed Cover Image Cover.tif}

